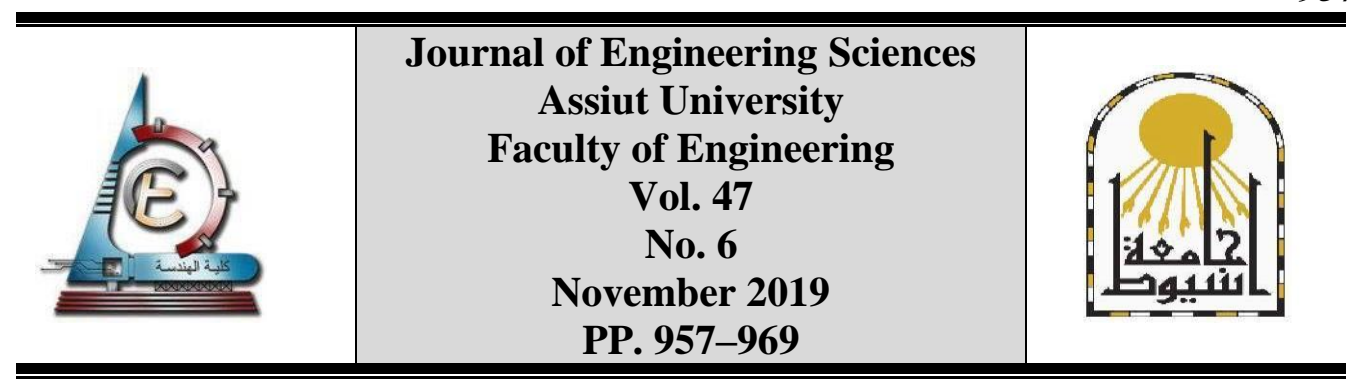

\title{
A GIS-BASED AUTOMATED ARCHITECTURAL SPATIAL ANALYSIS AND REPRESENTATION IN THE CONCEPTUAL DESIGN PHASE
}

\author{
Ayman Mohamed Assem \\ Department of Architecture, Faculty of Engineering, Ain Shams University; E- mail \\ address: ayman.assem@eng.asu.edu.eg
}

Received 5 November 2019; Accepted 21 November 2019

\begin{abstract}
This paper introduces an automated workflow using GIS model-builder that enhance the traditional conceptual design process using freehand sketching and CAD software packages and using other software packages for area calculations and visual presentation. Repeating the process of functional evaluation after receiving feedbacks from the clients is one of the main disadvantages of the traditional method, and could consume more time to reproduce calculation sheets and the graphical illustrations.

Accordingly, there is an obvious demand to save time and money and to enhance this process. However, using a third-party application could affect that process positively by providing an automated tool that generates a real-time calculation and produces automated graphical studies and colored illustrations.

Five steps should be performed to run the automated workflow using GIS model-builder. The original CAD file should be prepared before connecting to GIS then the CAD data sets will be extracted automatically to GIS geodatabase in a predetermined working folder, then sequential geoprocessing tools are run, starting with creating the new feature classes, and finally, the auto generated calculation sheets and colored studies and architectural plans come out.
\end{abstract}

Keywords: Geographic information system (GIS) - model builder - Spatial Analysis Conceptual Design - Architectural Representation

\section{Introduction}

There is no doubt that the conceptual phase of the architectural design is the most essential phase in the design process. Traditionally, freehand sketching is commonly used during the conceptual phase, because it gives the designer the ability to test out his ideas in a cheap and quick way. [1] 
Generally, using BIM in the design process provides designers with an opportunity to test the quality of the design and to find a suitable way to enhance the design products. However, using BIM in the first stage of the architectural design is considered the weakest side of using it. [2] One of the main reasons for not using BIM model from the beginning of the design process, is that it requires accuracy in modelling from the early beginning, and to get the best use of a BIM system, a complete BIM model should be created, which means that it is very difficult to prepare conceptual drawings in the early design stage. [3]

Accordingly, CAAD is commonly used in the design phase, and according to the designer's perspective, using CAAD systems has less risk than using BIM, especially in the conceptual phase. [4] Based on real life data using CAAD systems in the early design stages, CAAD is still faster than using BIM in the conceptual design and presentation stage. [4]

GIS with its various applications is involved in many design scales. It is commonly used in urban planning and urban design. However, currently GIS is used in architectural indoor analysis to take advantage of its power in storing, quarrying, analysing, and displaying all types of spatial data. Currently, GIS system is being developed to host all BIM data, and produce indoor maps derived from BIM models [5] . It is not surprising that many of the GIS leaders are architects. Currently, GIS is becoming relevant to architects because of using the concept of graphical overlay technique, which could be used in many architectural design phases. [6]

\subsection{Methodology}

This paper explores the potential of using the analytical capabilities of GIS in the spatial analysis of the architectural design, and extracting real-time spatial data, in addition to creating representations of architectural plans automatically. There's a variety of GIS software applications and packages, Arc-Info from ESRI is selected for the study because it includes all needed functionalities [7], especially the Model-Builder application which is used to create, edit, and manage workflows and sequences of geoprocessing modules. Model-Builder links other applications with ArcGIS and connects the outputs of one module or application into another one as input [8]. This workflow could be repeated many times by defining the parameters of the tool which are the AutoCAD file name, the folder path and the name of the new created GIS geodatabase.

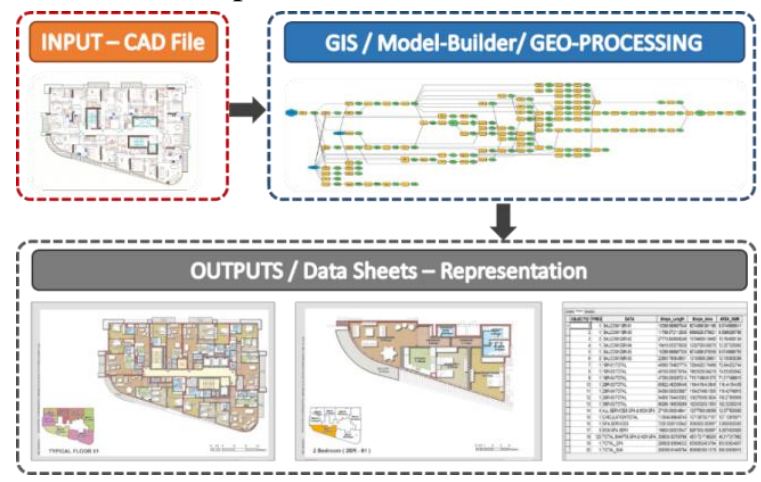

Fig. 1. The main workflow showing inputs and outputs using GIS model-builder. 


\section{Enhancing the conceptual design phase using GIS geoprocessing during the traditional workflow}

Using freehand sketches is considered the main start of any architectural concept. However, examining the quality of design and all of its functional issues is highly necessary during this phase. Functional issues include not only the calculation of areas such as the built-up area, the gross floor area and the leasable area, but also the quality of the design of spaces such as the spatial relations the arrangement of the furniture.

In order to carry out a quick examination of the conceptual design, many software packages are used separately. AutoCAD or any similar software app is used regularly for drafting and calculating the shape properties, M.S Excel or any similar software for advanced calculations, and Photoshop or any similar software for producing colored plans and illustrations.

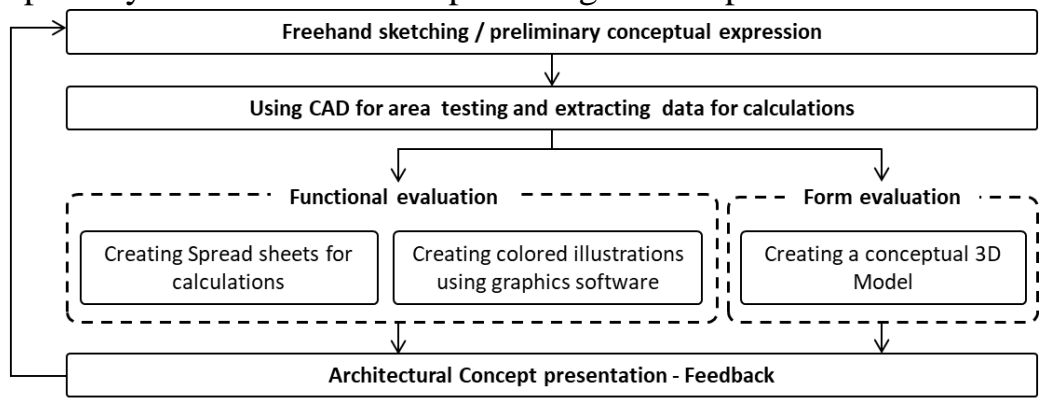

Fig. 2. The traditional workflow for the conceptual phase using AutoCAD and many other software

The previous figure (fig.2) illustrates the traditional workflow of the conceptual design phase influenced by using CAD software packages and using other software packages for area calculations and visual presentation. Repeating the process of functional evaluation after receiving feedbacks is one of the main disadvantages of the traditional method and could consume more time to reproduce calculation sheets and the graphical illustrations. However, using BIM software packages at this phase needs more time in the beginning of the project for preparation and creating families. Additionally, it is difficult to customize the calculation methods and the presentation styles based on the needs of that phase.

Accordingly, there is a real demand to save time and money and to enhance the functional evaluation process during the conceptual design phase. However, using a third-party application could affect that process positively by providing an automated tool that generates a real-time calculation and produces automated graphical studies and colored illustrations.

\subsection{Using GIS model-builder during the design conceptual phase}

One of the main advantages of using GIS software is that it can manipulate many types of data such as vector data or raster data or spread sheets or any other data types. The geoprocessing workflow can be automated using a GIS model-builder tool, which allows recording of the geoprocessing sequences and rerunning them many times later. 
Using the automated geoprocessing tool can provide the designer with an easy and fast tool that carries out a customized spatial calculation based on the required regulations and building codes, in addition to generating many types of colored illustrations with many predefined styles automatically.

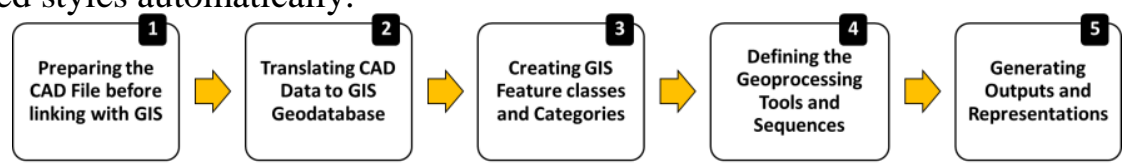

Fig. 3. The main five steps of the new automated conceptual design toolbox

Five steps should be performed to run the automated workflow using GIS modelbuilder. As shown in figure (3), the original CAD file should be prepared before connecting to GIS. Then the CAD data sets will be extracted automatically to GIS geodatabase in a predetermined working folder. The sequential geoprocessing tools are then run, starting with creating the new feature classes, ending up with the auto generated calculation sheets and colored studies and architectural plans.

In order to demonstrate the automated GIS geoprocessing, an example for a residential building has been selected to carry out the automated process.

\section{Step (1): Preparing the CAD File before linking with GIS}

In this step, a few essential actions for preparing the CAD file to be linked with the GIS automated toolbox are undertaken. GIS software can read specified types of vector data such as points, lines polygons and annotations, according to which the CAD file should be prepared form the beginning to be suitable for linking with the GIS geodatabase. During this step, three main actions regarding the definition and the arrangement of the CAD layers ensue.

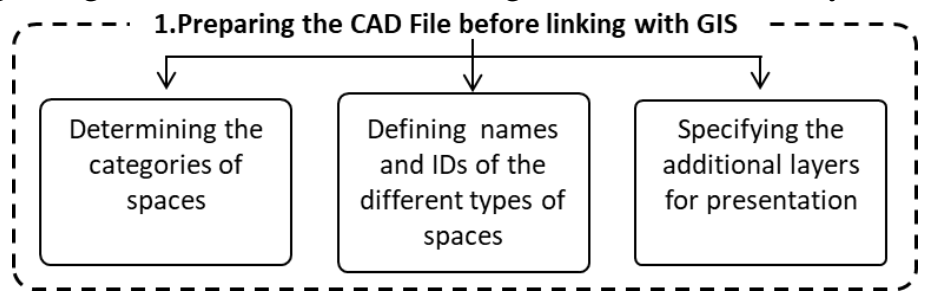

Fig. 4. The three actions of preparing the CAD file before linking to GIS

As shown in figure (4) the first action is to determine the different categories of spaces, the second action is to define the names and any other data related to spaces, and the last action is to specify any additional layers that could enhance the presentation of the generated colored illustrations. 


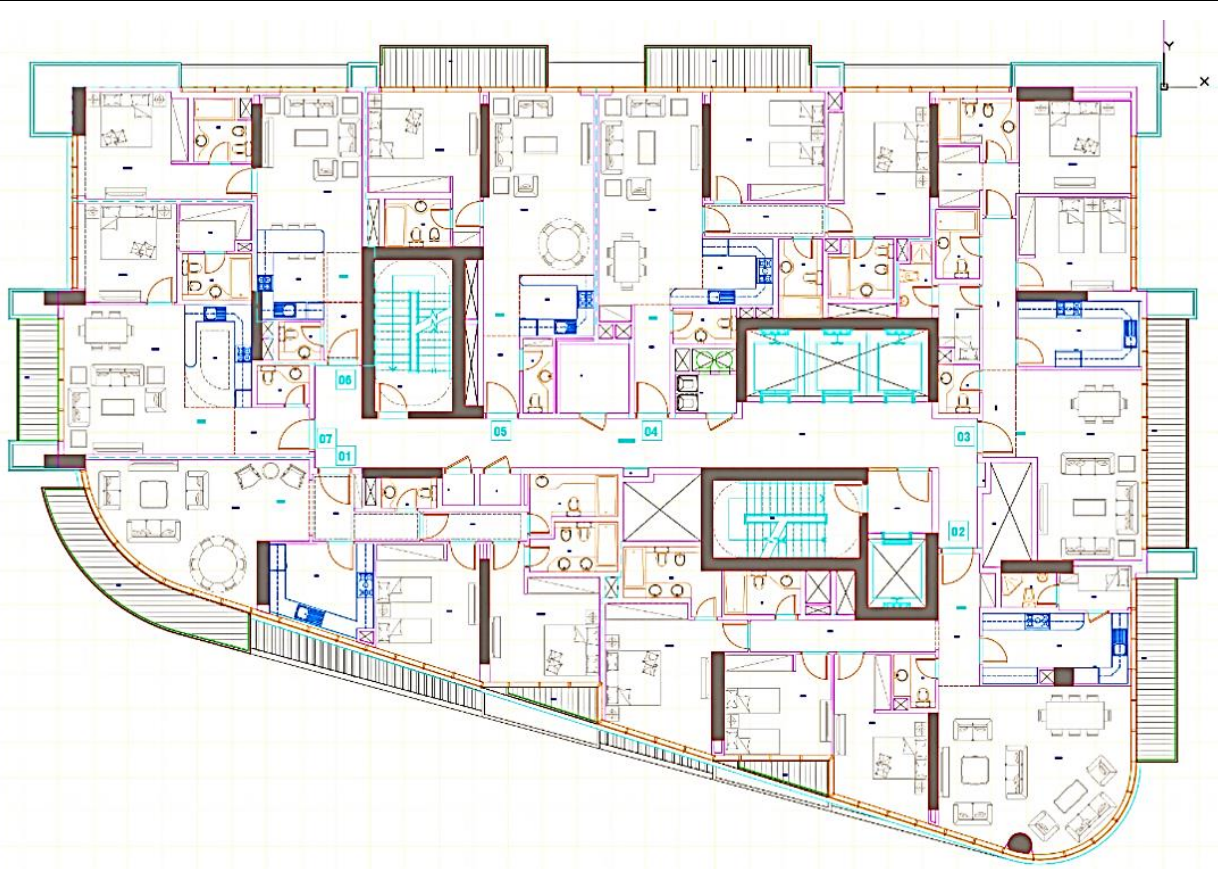

Fig. 5. The AutoCAD architectural plan showing different types of spaces and components

Figure (5) illustrates the original CAD file for the typical floor plan in the selected residential tower. The floor area is approximately 900 square meters and contains seven residential units in addition to the services, the public horizontal circulation area and the vertical circulation core.

\subsection{Determining the categories of spaces}

Spaces can be classified in different types or categories, based on their hierarchy and use. Each category should be defined in a specific layer and each space should be defined with a closed boundary. There is no need to define the boundaries as closed polylines or regions as GIS closes these shapes automatically with an error tolerance.

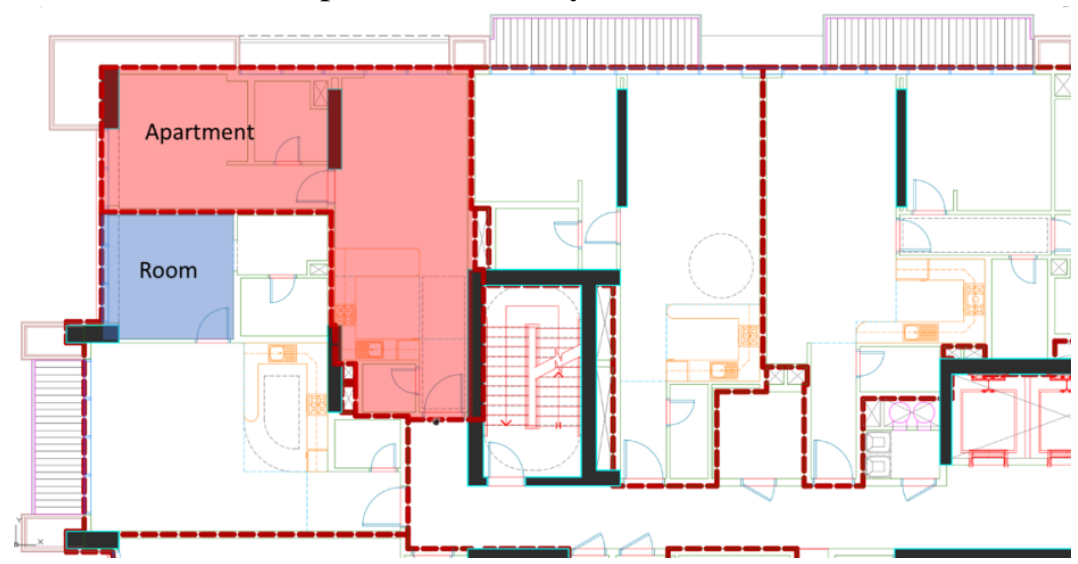

Fig. 6. A partial AutoCAD architectural plan showing different boundaries of spaces 
As shown in figure (6), the spaces could be categorized according to their hierarchy and usage as follows:
a. Floor boundary
b. Apartment boundary
c. Apartments Rooms
d. Room balcony
e. Services (defined with names)
f. Circulation spaces
g. Voids

\subsection{Defining names and IDs of the different types of spaces}

In all categories of spaces, each boundary of space should contain a unique name tag or ID. This data should be located approximately in the middle of the boundary and the text basepoint should be completely contained in the boundary of the space, as all that data will be defined in GIS as points containing different data fields. Each type of annotation should be placed in a separate layer related to the category of spaces.

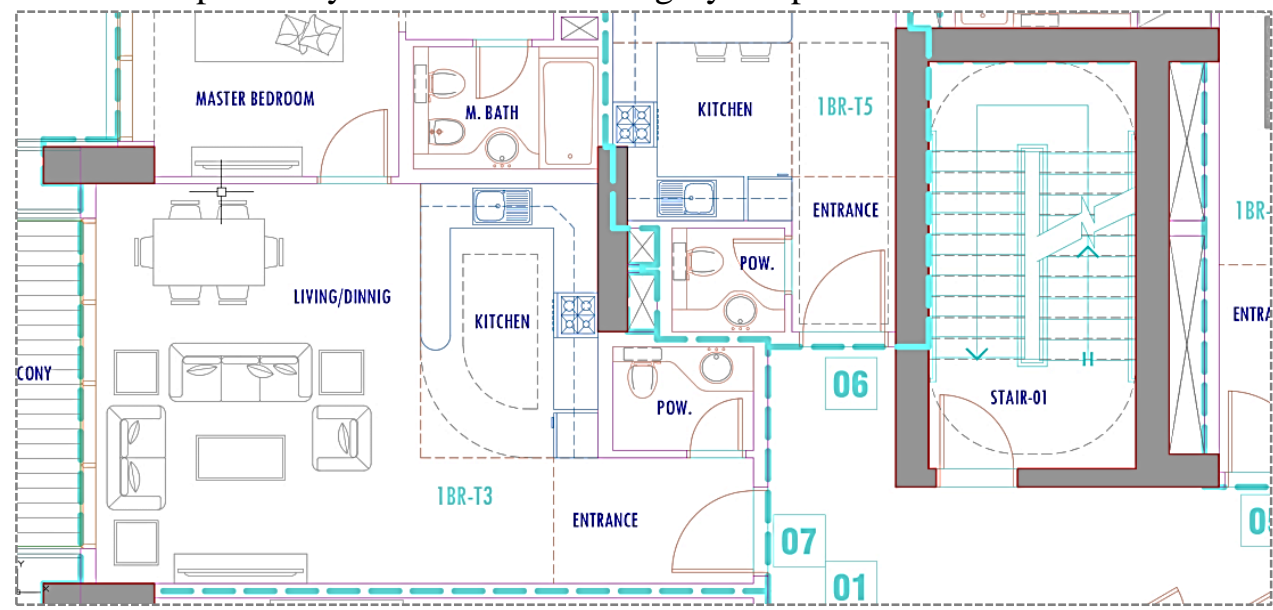

Fig. 7. A partial AutoCAD architectural plan showing the Definition of spaces

The previous figure displays a partial AutoCAD file for the residential floor, illustrating the different name tags and IDs contained in the different defined space boundaries, such as the IDs of the apartments, names of rooms and names of services. All these names and IDs will be connected according to their hierarchies using GIS tools.

\subsection{Specifying the additional layers for presentation}

To enhance the appearance of the presented plans and studies, many other layers should be extracted from the AutoCAD file to the GIS database. These data will be used in generating the raster datasets, witch, in turn, will be used in the colored illustrations such as wall layers, columns and structure elements, doors, windows, furniture elements, and all other architectural features. 


\section{Step (2): Translating CAD data to GIS geodatabase}

After preparing the AutoCAD file, the next step is to build the GIS database which will contain all types of extracted data and the new generated outputs. As shown in figure (8) this step is divided into two actions, the first is to create the working folder and the geodatabase, and the second action is to extract the AutoCAD data sets to GIS feature classes.

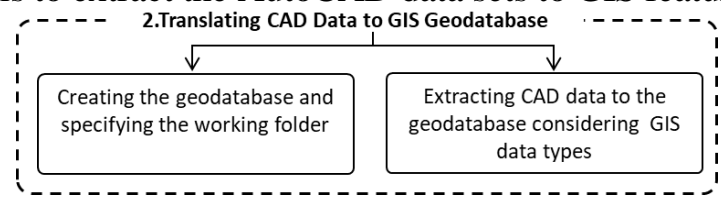

Fig. 8. The two actions for translating $\mathrm{CA} \overline{\mathrm{D}}$ data to match GiS data types

\subsection{Creating the geodatabase and specifying the working folder}

In order to manage the data extracted from AutoCAD and the data generated from the GIS model-builder, a new folder will be created automatically containing a new geodatabase, which is an alternate way to collect and store all GIS information in one big database, which contains all types of features such as points, lines polygons, and other data types like raster images and spread sheets.

The name of the folder and the geodatabase are defined as parameters in the new automated GIS tool, which means that the user will be asked to browse for the folder path and create the new geodatabase with a unique name.

\subsection{Extracting CAD data to the geodatabase considering GIS data types}

When extracting data from AutoCAD to GIS, it is necessary to consider the default feature classes that are used regularly in GIS. Many feature classes are recognized in GIS. However, only four types of GIS feature classes have been used in the new GIS conceptual tool. As shown in the figure (9), all extracted data should be translated to one of these four feature classes as Points, Polyline, Polygons and Annotations.

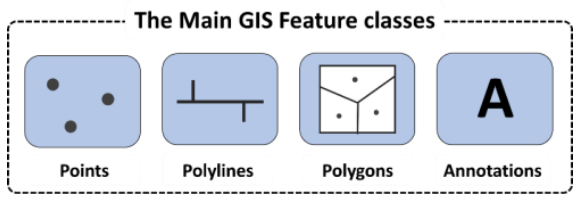

Fig. 9. The four feature classes used in the new GIS conceptual tool

After extracting the needed data from the AutoCAD file, all CAD data fields will be stored in the created GIS feature classes, such as the name of the layer, the color of objects and line weights. These data will be used for linking features and defining elements.

\section{Step (3): Creating GIS feature classes and categories}

In the previous step, the AutoCAD data sets have been translated into the main GIS feature classes, storing and keeping all original data extracted from the main AutoCAD 
Source file. Accordingly, these row feature classes should be reorganized and categorized based on the new workflow of the geoprocessing, to perform the required calculation and generate the needed colored illustrations.

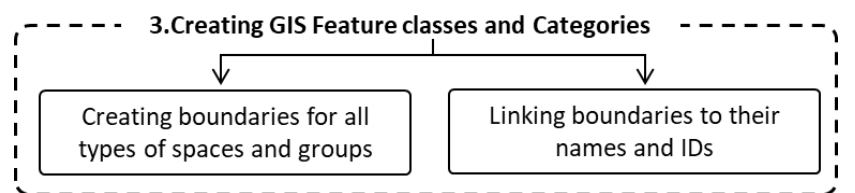

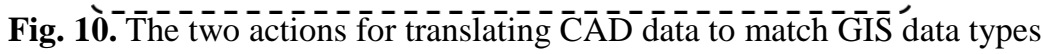

As shown in figure (10), two main actions should take place to create the new GIS analytical layers;

a. closing all boundaries of spaces,

b. Joining spaces with their information stored in another data type based on the spatial location of these information.

\subsection{Creating boundaries for all types of spaces and groups}

As mentioned in the previous step, all AutoCAD boundaries are extracted as lines. However, to determine the areas and the spatial properties of spaces, all boundaries should be defined as polygon feature classes. In order to perform this operation, a tool called 'polyline to polygon' is used to define the new polygons feature classes which will be used in the geoprocessing workflow.

\subsection{Linking boundaries to their names and IDs}

Based on the spatial relationship, the tool 'Spatial Join' joins attributes from one feature to another. The target features and the joined attributes from the join features are stored in a new output feature class [9]

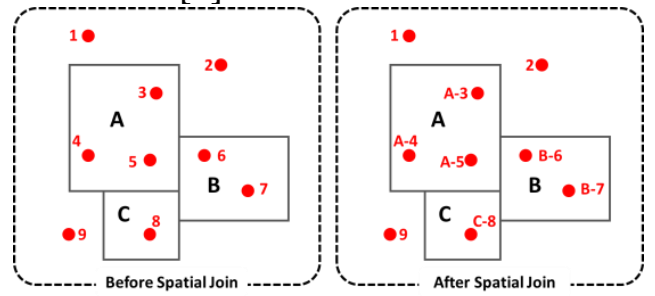

Fig. 11. The three actions of preparing the CAD file before linking to GIS

As shown in figure (11), a new feature class has been created after merging the data stored in the two features in the selected feature class, which is the point feature class in this example.

\section{Step (4): Defining the geoprocessing tools and sequences}

After linking all types of spaces with their names and IDs, the next step is to perform the geoprocessing workflow, which includes many intermediate geoprocessing tools that perform small operations, such as selecting spaces based on their names or IDs. 


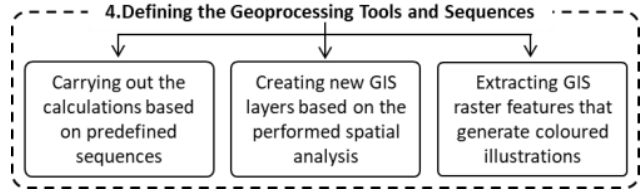

Fig. 12. The three actions of preparing the CAD file before linking to GIS

Figure (12) illustrates the three main actions taking place in this step:

a. carrying out the needed calculations,

b. create the intermediate layers,

c. extracting raster datasets.

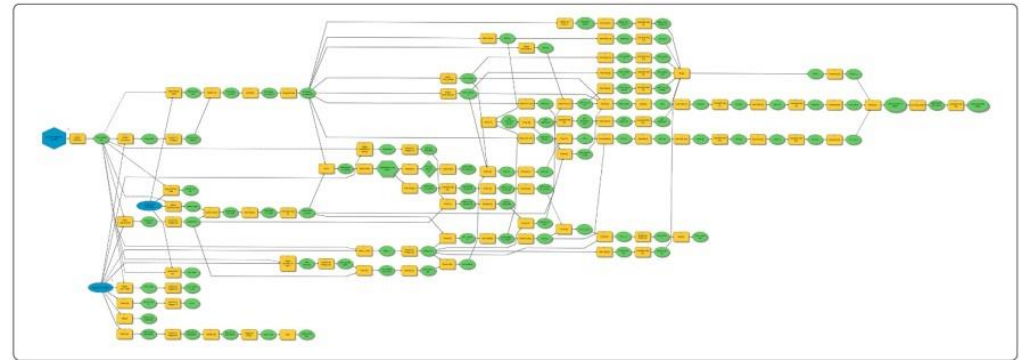

Fig. 13. The automated geoprocessing workflow using GIS model-builder

Figure (13) displays the automated geoprocessing workflow using GIS model-builder. The blue shapes indicate the input parameters coming from the AutoCAD file, the yellow shapes indicate the geoprocessing tools, and the green shapes indicate the output, which is connected to other tools as input.

Running the script takes, approximately, about three minutes for producing all spread sheets and illustrations.

\subsection{Carrying out the calculations based on predefined sequences}

Based on the GIS tools box, many tools can be used to extract the data from the generated feature classes using calculating tools, such as 'Frequency' which counts the number or calculates the area based on one or more reference.

\subsection{Creating new GIS layers based on the performed spatial analysis}

Many new GIS layers have been created as intermediate layers, using several geoprocessing tools such as follows:

- Select: selects features based on a predefined criterion

- Union: unifies two or many features from the same type in a new feature class

- Erase: erases selected features based on a predefined criterion

- Dissolve: merges similar features based on a predefined criterion 


\subsection{Extracting GIS raster features that generate coloured illustrations}

After creating the colored layers, the used layers for presentation could be converted to a raster layer by using the tool (Polygon to Raster). The converted layers will be used for generating the colored plans and illustrations. During the running of the geoprocessing workflow, a predefined color scheme will be loaded and assigned to the selected layers.

The produced layers have been stored in the created geodatabase, and will be added to the created MXD templates, based on the sequence of the workflow.

\section{Step (5): Generating Outputs and Representations}

The final step is to generate the calculation data sheets and all kinds of colored illustrations. As shown in the following figure there are three main actions in this step:

a. generating all calculated data as spreadsheets,

b. comparing the generated data with the original codes, regulation or guidelines,

c. Producing all colored plans and studies.

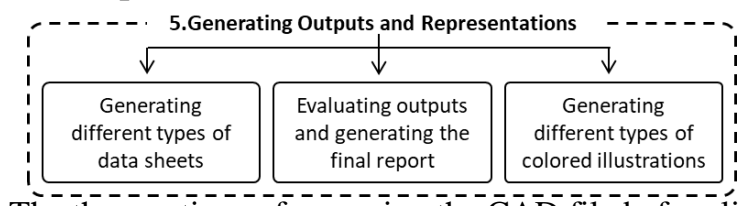

Fig. 14. The three actions of preparing the $\overline{\mathrm{C}} \overline{\mathrm{A}} \overline{\mathrm{D}}$ file before linking to GIS

\subsection{Generating different types of data sheets}

Based on the local regulation of the residential building and the requirements of the client, there are many ways of area calculations such as:

- BUA: calculating all built up areas including services and vertical cores

- GFA: calculating the gross floor areas including all apartments, and excluding parking, mechanical rooms and some types of services like electrical rooms

- $N L A$ : calculating the net leasable area, which is very important for the client to make his marketing study,

- The breakdown of rooms areas in all apartments

- The mix of the apartments based on the number of the bedrooms (1BR-2BR-3BR) and the number of keys,

- The area and the percentage of the horizontal circulations

- The net and the gross areas for the apartments 
Ayman mohamed assem, A GIS-based automated architectural spatial analysis and
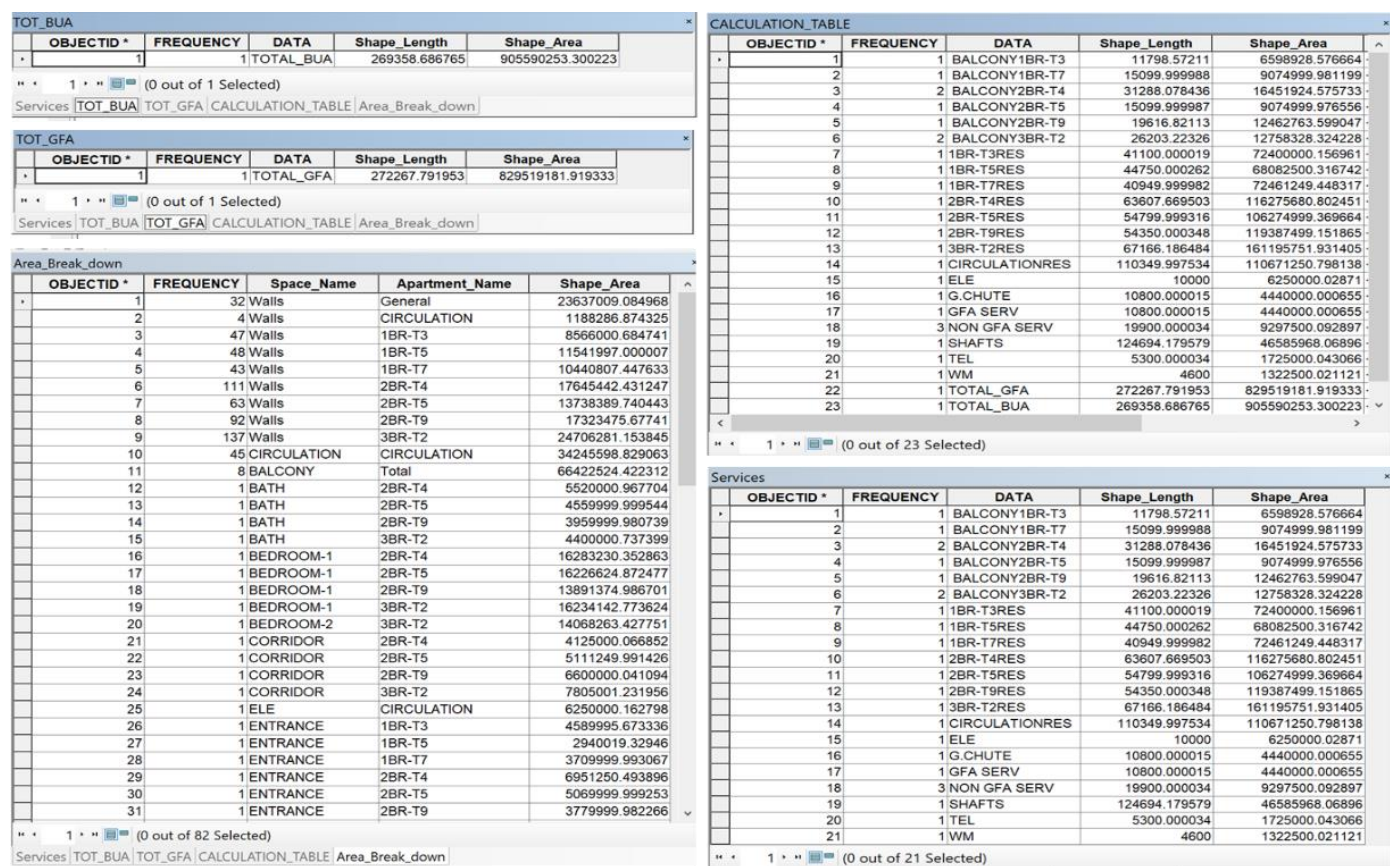

Fig. 15. The automated produced data sheets showing different types of calculations

The previous figure displays different generated spreadsheets that could be exported directly to an autogenerated report. Carrying out the area calculations is a customizable operation, and could be changed based on any new requirements.

Many other calculations have been generated, based on the spatial analytical power of the GIS, such as the following:

- The distances between two or many spaces.

- The buffer zone for a defined central point

- The visual studies in the indoor spaces like Isovist or Space syntax analyses

- Using topology to detect any unconsidered shape rules.

\subsection{Evaluating outputs and generating the final report}

After extracting the calculated datasheets, all extracted data could be compared with a predefined table that contains the original data written from the building codes or the local regulations or guidelines. A checklist could be autogenerated, describing how the generated data matched to the original regulations or codes.

\subsection{Generating different types of coloured illustrations}

Collecting and organizing the generated raster data and the colored GIS layers in an MXD template file, a set of drawings could be produced. In the MXD file, the same data could be displayed several times with several presentation styles. The data frames could be 
presented in different scales in the same sheet. The produced drawing sheets could be exported automatically with different sizes or different resolutions as needed.

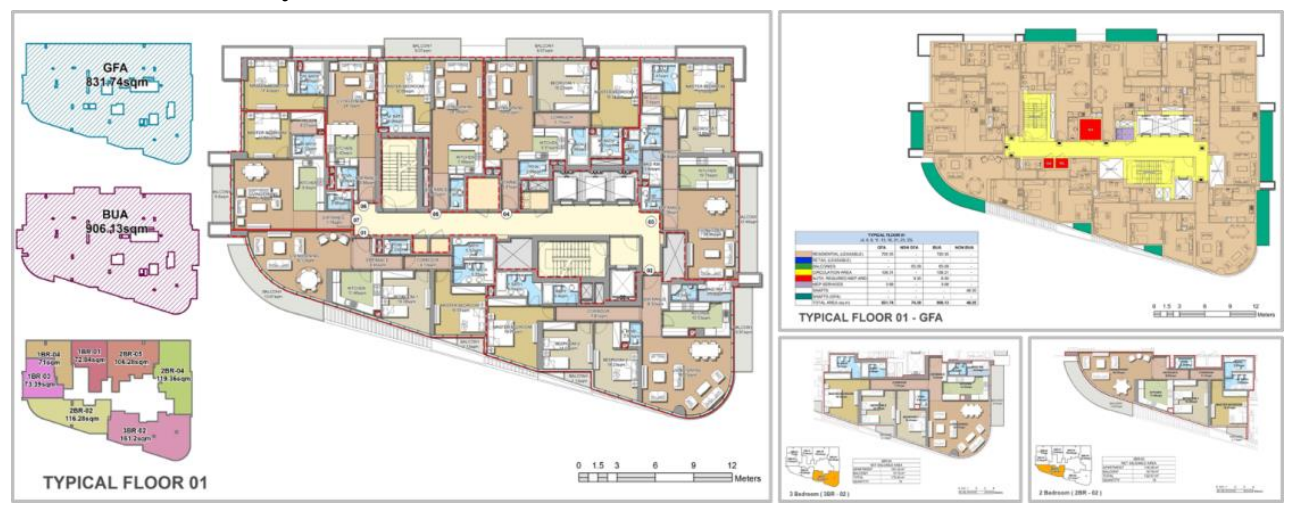

Fig. 14. The automated generated plans showing different types of illustrations and details

In the selected example, many types of illustrations have been generated automatically, such as the following:

- Colored plans with different color themes.

- Space use study defining different types of spaces

- BUA and GFA studies

- Enlargement plans for each apartment with more detailed furniture.

- Key plans for each apartment and each floor

\section{Conclusion}

- Using GIS model-builder to devise a third-party automated application could enhance the conceptual design phase by providing the designers with fully customizable applications that allow adaptation based on design codes, local guidelines and client requirements. That automated process could be repeated hundreds of times in a very short duration, and provides the potential for real-time design evaluation and validation.

- Although there are obvious benefits of using a third-party application for enhancing the traditional conceptual design phase, there are many difficulties of using GIS with this regular process. However, it could be better if the application is developed into a standalone software interface, or a web-based application that could be run through a GIS server.

- Converting the design freehand sketches directly to the analytical tool, without translation to CAD then GIS, is a big challenge in the future, especially, with the current rapid development in scanning and image processing.

\section{REFERENCES}

[1] T. D. a. G. D. P. Yi Zhu, "A Comparing Study of the influence of CAAD Tools to Conceptual Architecture Design Phase," in EuropIA'11: 11th International Conference on Design Sciences and Technology, Montreal , 2007.

[2] Ö. H. Çavuşoğlu, "The Position of BIM Tools in Conceptual Design Phase: Parametric Design and Energy Modeling Capabilities," in eCAADe 33, Wien, 2015. 
[3] A. P. Ireneusz Czmocha, "Traditional Design versus BIM Based Design," in XXIII R-S-P seminar, Theoretical Foundation of Civil Engineering (23RSP) (TFoCE 2014), 2014.

[4] D. Holzer, "BIM's seven deadly sins," International Journal of Architectural Computing, no. December , 2011.

[5] B. L. S. Z. H. L. L. Liu, "THE PATH FROM BIM TO A 3D INDOOR FRAMEWORK - A REQUIREMENT ANALYSES," in The International Archives of the Photogrammetry, Remote Sensing and Spatial Information Sciences, Volume XLII-4, The Netherlands, 2018.

[6] M. Z. I. Muntazar Monsur, "GIS for Architects:Exploring the Potentials of Incorporating GIS in Architecture Curriculum," in ARCC/EAAE 2014 INTERNATIONAL ARCHITECTURAL RESEARCH CONFERENCE , Honolulu, Hawaii U.S.A., 2014.

[7] "ArcGIS Pro," [Online]. Available: https://pro.arcgis.com/en/pro-app/tool-reference/3danalyst/comparing-interpolation-methods.htm. [Accessed 22 May 2019].

[8] "ArcGIS for Desktop - What is ModelBuilder?," ESRI, [Online]. Available: http://desktop.arcgis.com/en/arcmap/10.3/analyze/modelbuilder/what-is-modelbuilder.htm. [Accessed 10 October 2019].

[9] "ArcGIS for Desktop - $\quad$ Spatial Join," ESRI, [Online]. Available: http://desktop.arcgis.com/en/arcmap/10.3/tools/analysis-toolbox/spatial-join.htm. [Accessed 1110 2019].

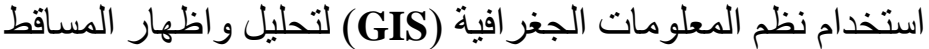 \\ و الفر اغات المعمارية بطريقة أوتوماتيكية في مرحلة إعداد الفكرة التصميمية}

\section{الملخص العربى}

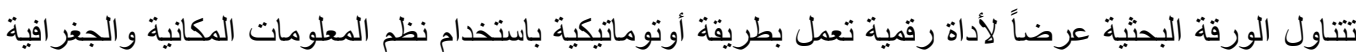

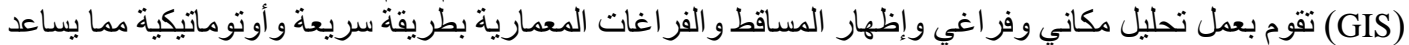

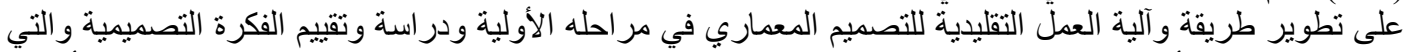

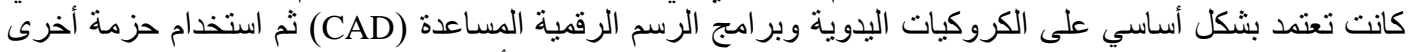

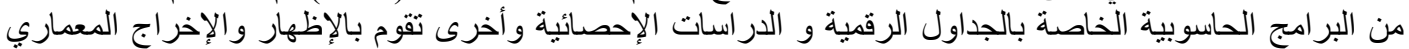

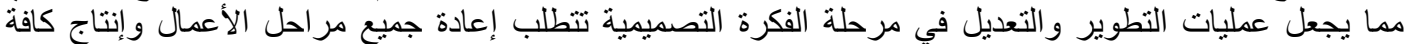

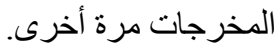

تهدف فكرة آلية العمل المستحدثة بشكل أساسي إلي توفير الوقت و الجهد في مرحلة إعداد الفكرة التصميمية وذلك

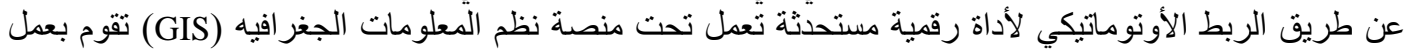

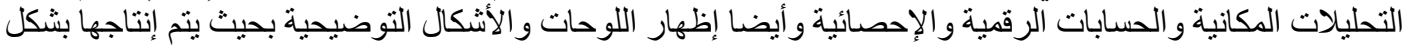

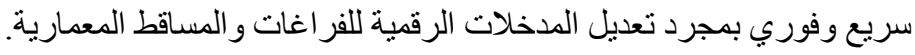

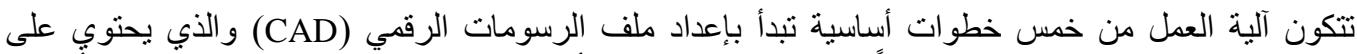

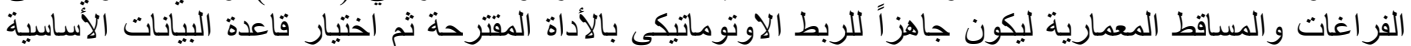

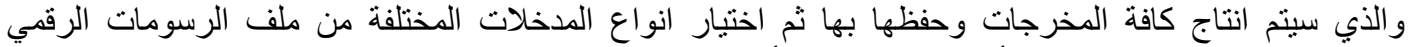

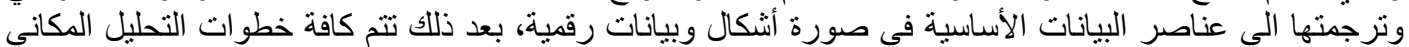

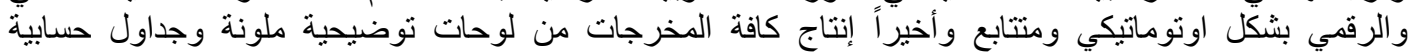

